\title{
Android Application on Creating Awareness on Illegal Activities in Forest
}

\author{
R. Jyothi Sulochana, R. Bindhu, R. Hema and M. Lorate Shiny
}

\begin{abstract}
Smuggling of trees like sandal, "Sagwan" etc have created a highlight in many newspapers. There is scarcity of these trees in world thus are very costly. These are used in the medical sciences as well as cosmetics. Because of huge amount of money concerned in selling of such tree woods lots of incidents are happening of their smuggling In India and also in the jungles of Karnataka and Tamilnadu. To restrict such smuggling and to save the forests around the globe some preventive measures need to be implemented. We are developing such a system which can be used to restrict this illegal business. The suggested system will consist of three different modules as follows, A. Tree Unit, B. Area unit and C. Main Server Unit. Every tree will be outfitted with one small electronics unit which consists of Micro Controller,3 Sensors and RF module. There will be one sub server unit for fussy area of jungle
\end{abstract}

Keywords--- Android Phone, GSM Modem, LCD, Max 232 Microcontroller, Main and Sub Server Unit, RF Module, Sensors, Tree Unit.

\section{INTRODUCTION}

We are on the rise such a system which can be used to restrict the illegal business of the trees which will stop the deforestation to solve one of the issues with the Global Warming. The system consisting of three stages:
i. Tree unit
ii. Sub-server unit
iii. Main server unit

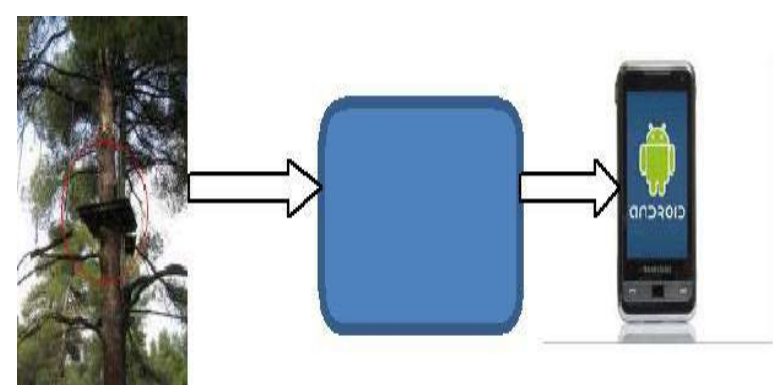

Figure 1.1

R. Jyothi Sulochana, Student, Dept of CSE, SSEC, Bangalore, India. E-mail:jyothijyo7207@gmail.com

R. Bindhu., Student, Dept of CSE, SSEC, Bangalore, India. E-mail:bindhunaidu96@gmail.com

R. Hema, Student, Dept of CSE, SSEC, Bangalore, India. E-mail:hema.raju01@gmail.com

M. Loret Shiny, Asst Professor, Dept of CSE, SSEC, Bangalore, India. E-mail:maxlinshiny@gmail.com

DOI:10.9756/BIJSESC.8233
Tree Unit

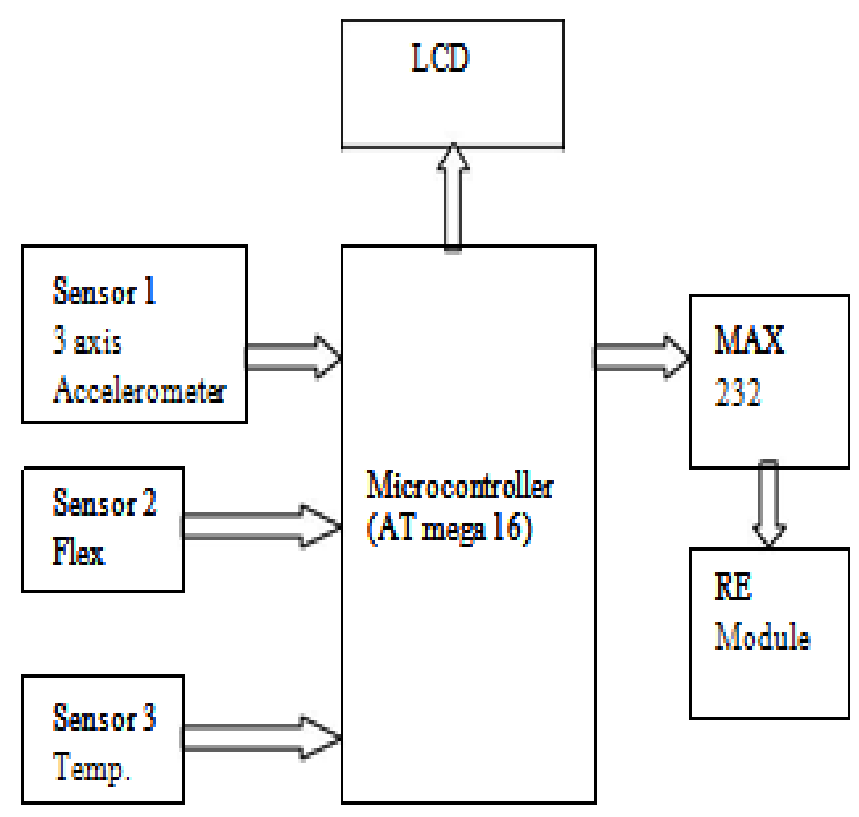

Figure 1.2: Tree Unit

The tree unit consists of three sensors:

a. 3 Axis Accelerometer Sensor

b. Flex Sensor

c. Temperature Sensor

The information to the micro-controller are sent by these sensors. To the tree unit which would be then transmitted to the next stage which is, Sub Server Unit, for further processing.

\section{Sub Server Unit}

This is the $2^{\text {nd }}$ stage of the system which would be responsible for gathering and facilitating the data to the MainServer Unit.

The Sub server unit is in charge to host the information from multiple Tree Units.

Thus in the real time scenario we can categorize multiple Sub-Server Units as one 1 Sub-Server Unit for every few Kilometers. 


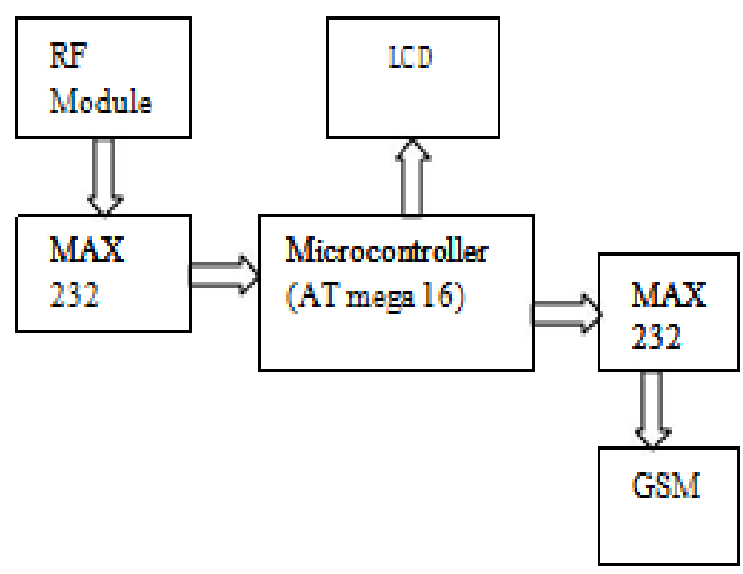

Figure 1.3: Sub Sever Unit

The Sub-Server unit contains RF module, Max 232 and the Micro-Controller which are responsible for the data transmission from Primary stage to the Final Stage of the Project.

\section{Main Server Unit}

This is the Final Stage of the system which is in command intended for the user interface and displaying the data that was transmitted from the step 1 and step 2. Main server would consist of these multiple things:

1. Storage Device

2. GSM Modem

3. Micro-Controller

4. An android Device.

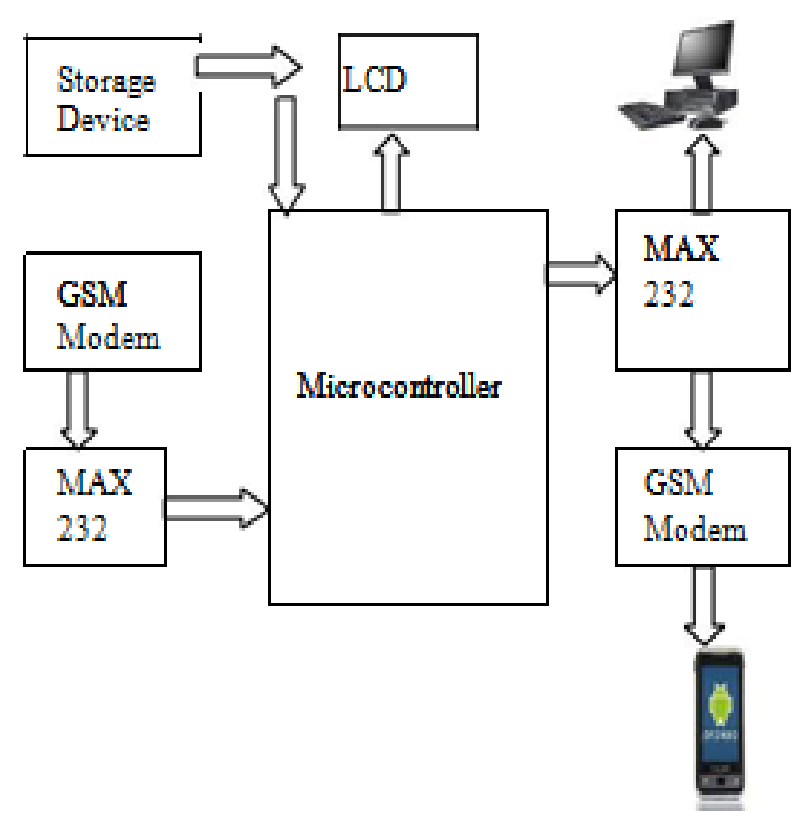

Figure 1.4: Main Sever Unit

The GSM modem would be fetching the data from the Micro-Controller and driven it to the Max232 which would have the configuration to send the data to machine Device. If the machine device is not easy to get the same which would be redirected to the configured System.

\section{SYSTEM SETUP}

\section{A. Three s Axis Accelerometer}

An accelerometer is an electromechanical device which measures the speeding up forces. These forces may be stationary like the constant force of gravity pulling at our feet, or they could be energetic - caused by moving or vibrating the accelerometer. Hypothetically an accelerometer behaves as a damped mass on a spring. When the accelerometer experiences an speeding up the mass is displaced to the point that the spring is able to accelerate the mass at the same rate as the casing. The dislocation is then measured to give the acceleration

Here MEMS 3 axis accelerometer is used. which consists of a accumulation at the sensor's chip, which is balanced by 4 beams doped with piezo resistive substance When the sensor is subjected to acceleration instant of gathering causes the 4 beams to cave in and so change the resistance in piezo substance which enables the sensor to detect the acceleration action

Fujitsu's small and highly aware accelerometer are used to detect acceleration inclination and vibration by measuring the motion in the X-, Y-, and Z-axis concurrently

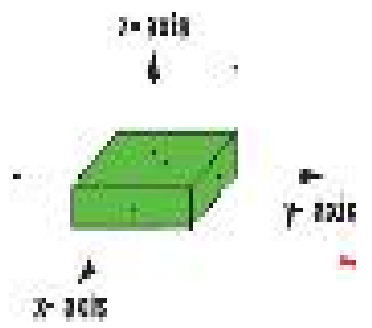

Figure 2.1: Principle of Detection

\section{B. Flex Sensor}

Flex sensors are inactive resistive devices that can are used to identify flexing or bending. Flex sensors are sensors that change in resistance depending upon the quantity of bend on the sensor which is contained by the flex sensor constitute carbon resistive elements, within a thin elastic substrate, boost the content of carbon decreases resistance. The flex sensor implemented here is a one-directional flex sensor that decreases its resistance in proportion.
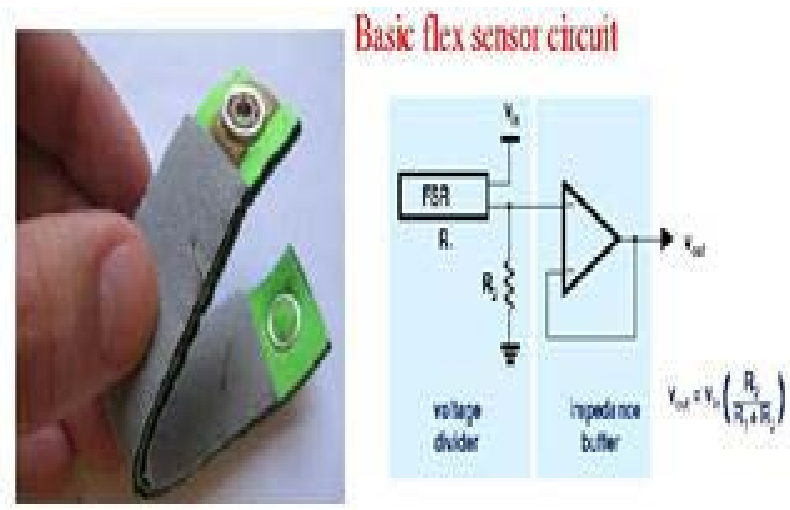

Figure 2.2: Flex Sensor 


\section{Temperature Sensor}

Temperature sensor are used to sense the disaster of fire and hence to save the trees from getting blistered due to fire. Temperature sensor used is LM 35.

The LM35 sequences are precision integrated-circuit temperature sensors, whose output voltage is linearly related to the Celsius temperature. The LM35 thus has an advantage in excess of linear temperature sensors calibrated in ${ }^{\circ}$ Kelvin, as the user is not required to subtract a large constant voltage from its output to obtain suitable Centigrade scaling.

\section{Android Phone}

An android phone is a cell phone that uses the Google urbanized Android operating system and stage. The phone is Linux kernel-based, has functions that are written in the Java language and can still run applications written in the $\mathrm{C}$ language. Google united with the High Tech Computer Corporation (HTCC) to construct hardware for the G1 cell phone, which was the first to run the Android platform.

\section{DATA PRocessing}

\section{A. Data Transmission}

3 axis accelerometer is used to sense motion of tree in all three coordinates $\mathrm{x}, \mathrm{y}$ and $\mathrm{z}$. Flex sensor is used to detect angle Temperature sensor is used for protection in case of fire. All three signals are given to microcontroller AT mega16. Output of AT mega 16 is provided to Max 232. If the tree is getting cut or if there is misfortune of fire, then these 3 sensors will give signal to microcontroller AT mega 16 . There is no need of ADC in between sensors and microcontroller because AT mega 16 has inherent ADC. Max 232 gives that signal to RF module. At sub server unit signals are accepted through RF module again.Received signals are given to microcontroller all the way through max232. Output signal from microcontroller is given to GSM through max 232 again and then this signal is transmitted to main server unit.

Sub server unit is used connecting tree unit and main server unit because if tree is getting collapsed then tree unit will be offended but we will have the data in sub server unit, which tree is getting collapsed, exactly which place etc.

\section{B. Data Reception}

Storage device could be a server with multiple storage drives or a disk capacity Signals from sub server unit are received here. Level converter max 232 gives the received signal to microcontroller. Microcontroller gives that signal to PC through

Max232.we can directly have the received data on smart phone all the way through GSM.

\section{CONCLUSION}

In this paper, we have proposed an application in android to create an awareness of smuggling trees in forest. We performed an experiment to determine the extent to which these type of smuggling can be automatically detected from data processing. The experiment shows that our approach outperforms the audit strategy of ANTI SMUGGLING of trees. Therefore we conclude that our approach is a substantial improvement over these illegal activities.

\section{REFERENCES}

[1] I. Lee, G.H. Yoon, J. Park, S. Seok, K. Chun and K. Lee, "Development and analysis of The vertical capacitive accelerometer", Sensors and Actuators A: Physical, Vol. 119 No. 1, Pp. 8-18, 2005.

[2] F. Chollet and H. Liu, "short introduction to MEMS", (http://memscycl opedia.org.introMEMS.html (18.2.2008)).

[3] S. Beeby, G. Ensell, M. Kraft and N.M. White, "MEMS mechanical sensors", Artech house inc.

[4] S.E. Lyshevski, "MEMS and NEMS:systems, devices and structures", CRC Press LLC, USA.

[5] Allen B. Downey, "Changepoint Detection in network measurements", Franklin W. Olin College of Engineering.

[6] S. Chen, J. Wang and D. Wang, "Extraction of fuzzy rules by using support vector machines", Fifth International Conference ON Fuzzy Systems and Knowledge Discovery, Vol. 3, Pp. 438-442, 2008.

[7] H. Cheng, C. Potter and S. Klooster, "Detection and characterization of anomalies in multivariate time series”, SDM, Vol. 9, Pp. 413-424,2009.

[8] C. Jensen and C. Potts, "Privacy practices of internet users: self-reports versus observed behavior", International Journal of Human Computer Studies, Vol. 63, No. 1, Pp. 203-227, 2005.

[9] C. Herley, "So. Long and No Thanks for the Externalities: The Rational Rejection of Security Advice by Users", Proceedings of the 2009 workshop on New security paradigms workshop, Pp.133-144, 2013. 